\title{
SOME ANALOGS OF THE GENERALIZED PRINCIPAL AXIS TRANSFORMATION
}

\author{
N. A. WIEGMANN
}

It is known that two normal matrices can be diagonalized by the same unitary transformation if and only if they commute; this theorem is ordinarily stated for hermitian matrices. Some generalizations of this theorem are known. According to a theorem due to Eckert and Young, ${ }^{1}$ if $A$ and $B$ are two $r \times s$ matrices, there are two unitary matrices $U$ and $V$ such that $U A V=D_{1}$ and $U B V=D_{2}, D_{1}$ and $D_{2}$ diagonal matrices with real elements, if and only if $A B^{c t}$ and $B^{c t} A$ are hermitian. It is also known that a set of normal matrices $\left\{A_{i}\right\}$ is reducible to diagonal matrices under the same unitary similarity transformation, $U A_{i} U^{c t}$, if and only if $A_{i} A_{j}=A_{j} A_{i}$ for all $i$ and $j$. (More generally, it is true that a set of matrices $\left\{A_{i}\right\}$ with elements in the complex field and simple elementary divisors is reducible to diagonal matrices under the same similarity transformation if and only if $A_{i} A_{j}=A_{j} A_{i}$ for all $i$ and $j$.) The following will be shown to hold:

THEOREM. If $\left\{A_{i}\right\}$ is an arbitrary set of nonzero $r \times$ s matrices, there are unitary matrices $U$ and $V$ of orders $r \times r$ and $s \times s$, respectively, such that $U A_{i} V=D_{i}, D_{i}$ diagonal and real, if and only if $A_{i} A_{j}^{c t}=A_{j} A_{i}^{c t}$ and $A_{j}^{c t} A_{i}=A_{i}^{c t} A_{j}$ for all $i$ and $j$.

If two unitary matrices $U$ and $V$ exist such that $U A_{i} V=D_{i}, D_{i}$ real for all $i$, then $D_{i} D_{j}^{c t}=D_{i} D_{j}^{t}=D_{j} D_{i}^{t}=D_{j} D_{i}^{c t}$ where the $D_{i}$ are $r \times s$ diagonal matrices (that is, the only nonzero elements appear in the $d_{i i}$ position). Therefore, $A_{i} A_{j}^{c t}=A_{j} A_{i}^{c t}$.

Conversely, let the relations $A_{j}^{c t} A_{i}=A_{i}^{a t} A_{j}$ and $A_{i} A_{j}^{c t}=A_{j} A_{i}^{c t}$ hold for all $i, j$. The proof is by induction.

(1) The theorem is true for a set of matrices of dimension $1 \times s$, $A_{i}=\left[a_{i}^{\prime}, a_{i}^{\prime \prime}, \cdots, a_{i}^{(s)}\right]$. For there exist unitary matrices $U$ and $V$ such that ${ }^{1} U A_{1} V=\left[d_{1}^{\prime}, 0, \cdots, 0\right]$ for $d_{1}^{\prime}$ real and greater than 0 since $A_{1} \neq 0$. For if $U A_{i} V=\left[d_{i}^{\prime}, d_{i}^{\prime \prime}, \ldots, d_{i}^{(s)}\right]$, it follows from $A_{i}^{c t} A_{1}=A_{1}^{c t} A_{i}$ that $d_{i}^{\prime \prime}=d_{i}^{\prime \prime \prime}=\cdots=d_{i}^{(s)}=0$ and since $d_{1}^{\prime} \cdot \bar{d}_{i}^{\prime}=\bar{d}_{1}^{\prime} \cdot d_{i}^{\prime}$ and $d_{1}^{\prime}$ is real, $\bar{d}_{i}^{\prime}=d_{i}^{\prime}$. In the same way by means of the second of

Presented to the Society, April 26, 1947; received by the editors November 26, 1947.

1 Bull. Amer. Math. Soc. vol. 45 (1939) pp. 118-121. See also J. Williamson, Bull. Amer. Math. Soc. vol. 45 (1939) pp. 920-922. 
the given conditions, the theorem is true for a set of matrices of dimension $r \times 1$.

(2) Assume the theorem to be true for a set of matrices of dimension $k \times l$ for $k \leqq r, l \leqq s-1$ and for $k \leqq r-1, l \leqq s$. The theorem will be shown to hold for the dimension $r \times s$ and the induction will then be complete. Let $\left\{A_{i}\right\}$ be a set of matrices of dimension $r \times s$ for which the given conditions hold. Let $U$ and $V$ be such that

$$
U A_{1} V=\left[\begin{array}{ll}
D & 0_{2} \\
0_{3} & 0_{4}
\end{array}\right]
$$

where $U$ and $V$ are unitary, $D$ a nonsingular diagonal matrix with real positive diagonal elements, and the submatrices $0_{2}, 0_{3}$, and $0_{4}$ are null matrices or non-existent. If

$$
U A_{i} V=\left[\begin{array}{ll}
G_{i} & K_{i} \\
L_{i} & H_{i}
\end{array}\right]
$$

it follows from $A_{1} A_{i}^{a}=A_{i} A_{1}^{a t}$ that $L_{i}=0_{3}$, and from $A_{i}^{a t} A_{1}=A_{1}^{c t} A_{i}$ that $K_{i}=0_{2}$. Also, $D G_{i}^{c t}=G_{i} D$ and $G_{i}^{c} D=D G_{i}$ from both given conditions. Therefore, $D^{2} G_{i}^{c t}=D G_{i} D=G_{i}^{c t} D^{2}$ so $D^{2} G_{i}=G_{i} D^{2}$. Since $D$ consists of positive real numbers and since $G_{i}$ commutes with $D^{2}, D G_{i}$ $=G_{i} D$. Then $D G_{i}^{a}=G_{i} D=D G_{i}$ and since $D$ is nonsingular, $G_{i}^{a}=G_{i}$ for all $i$. Then from the given relation $A_{i} A_{j}^{a}=A_{j} A_{i}^{a}$, it follows that $G_{i} G_{j}^{a t}=G_{j} G_{i}^{a}$ or $G_{i} G_{j}=G_{j} G_{i}$; therefore the set of hermitian matrices $\left\{D, G_{i}\right\}$ are all commutative in pairs and, by the generalized principal axis theorem, there exists a unitary matrix $U_{1}$ which diagonalizes all of them. Let $U_{2}=U_{1}+I$ be a unitary matrix of the same dimension as $U$ and $U_{3}=U_{1}^{a}+I$ of the same dimension as $V$. Then,

$$
U_{2} U A_{1} V U_{3}=\left[\begin{array}{cc}
D & 0_{2} \\
0_{3} & 0_{4}
\end{array}\right], U_{2} U A_{i} V U_{3}=\left[\begin{array}{cc}
D_{i} & 0_{2} \\
0_{3} & H_{i}
\end{array}\right]
$$

for all $i$ where the $H_{i}$ are either non-existent or of dimension $k \times l$ where $k<r$ and $l<s$. The theorem follows from the induction hypothesis.

It is to be noted that if the set $\left\{A_{i}\right\}$ are all $n \times n$ hermitian matrices for which $A_{i} A_{j}^{a}=A_{j} A_{i}^{a}$ or $A_{i} A_{j}=A_{j} A_{i}$ holds, the principal axis transformation for hermitian matrices is obtained and $V=U^{c t}$.

According to another result due to Eckert and Young, ${ }^{1}$ if $A$ and $B$ are $r \times s$ matrices over the complex field, a necessary and sufficient condition that there exist two unitary matrices $U$ and $V$ such that $U A V=D_{1}$ and $U B V=D_{2}, D_{1}$ and $D_{2}$ diagonal, is that $A B^{c t}$ and $B^{c t} A$ be normal. Since this is a generalization of the earlier result, it would 
seem reasonable to hope for an extension to a set of matrices $\left\{A_{i}\right\}$. A simple example shows that this is not the case, however, and the following theorem holds:

Theorem. $A$ necessary and sufficient condition that a set of $n \times n$ matrices $\left\{A_{i}\right\}$ be brought into diagonal forms by the same unitary $U$, $V$ equivalence transformation, $U A_{i} V=D_{i}$, is that the products $A_{i} A_{j}^{\text {ct }}$ and $A_{j}^{c t} A_{i}$ be normal for all $i, j$ and that $A_{k}\left(A_{j}^{a t} A_{i}\right)=\left(A_{i} A_{j}^{c t}\right) A_{k}$ for all $i$, $j$ and $k$.

If $U A_{i} V=D_{i}$ for all $i$, then the given conditions can be easily verified.

Conversely, let $\left\{A_{i}\right\}$ be a set of matrices for which $A_{i} A_{j}^{\text {at }}$ and $A_{j}^{c t} A_{i}$ are normal and where $A_{k}\left(A_{j}^{c t} A_{i}\right)=\left(A_{i} A_{j}^{c t}\right) A_{k}$. The proof is by induction on the order $n$. The theorem is trivially true if $n=1$. Assume it to be true for order $k \leqq n-1$. Now consider a system of order $n$. There are two possibilities: for all $i, j$, either $A_{i} A_{j}^{c t}$ is a scaler matrix or there is at least one pair $i, j$ such that $A_{i} A_{j}^{c t}$ is not a scaler.

(1) If for all $i, j, A_{i} A_{j}^{c t}$ is a scaler, $A_{i} A_{j}^{c t}=k_{i j} I$ and since $A_{i} A_{j}^{c t}$ is similar to $A_{j}^{c t} A_{i}$, it is true that:

$$
A_{i} A_{j}^{c t}=k_{i j} I=A_{j}^{c t} A_{i} \quad \text { for all } i, j .
$$

There are two possibilities: (a) Either all $A_{i}=k_{i} U_{i}$ where the $k_{i}$ are real positive scalers and $U_{i}$ are unitary; then all $A_{i}$ are normal and from the above, $A_{i} A_{j}=A_{j} A_{i}$ since $A_{j}=f\left(A_{j}^{c}\right)$. In this case the principal axis transformation theorem applies for normal matrices so $V=U^{c t}$ and the theorem is true. (b) There is at least one $A_{i}$, say $A_{1}$, not of the above form. There exist two unitary $U, V$ such that $U A_{1} V=D_{1}$ is diagonal with real non-negative elements. Also, $D_{1}$ is not scaler for then $A_{1}=U^{c t} D_{1} V^{c t}=D_{1} U^{c t} V^{c t}$; but this contradicts the assumption. Let $U A_{j} V=A_{j}^{\prime}$. Then,

$$
\begin{aligned}
& U A_{1} V V^{c t} A_{j}^{c t} U^{c t}=U k_{1 j} U^{c t}=k_{1 j} I=D_{1} A_{j}^{\prime c t} ; \\
& V^{c t} A_{j}^{c t} U^{c t} U A_{1} V=V^{c t} k_{1 j} V=k_{1 j} I=A_{j}^{\prime c t} D_{1} .
\end{aligned}
$$

Therefore,

$$
D_{1} A_{j}^{\prime c t}=A_{j}^{\prime c t} D_{1}
$$

and

$$
A_{j}^{\prime} D_{1}=D_{1} A_{j}^{\prime} \text {. }
$$

Since $D_{1}$ is not scaler, the $A_{j}^{\prime}$ are direct sums of matrices of order 
$k \leqq n-1$. But for these matrices the given conditions hold and the theorem is true.

(2) If for some $i, j$ the products $A_{i} A_{j}^{c t}$ (and consequently $A_{j}^{c t} A_{i}$ ) are not scaler, there exist for this $A_{i}$ and $A_{j}$ unitary matrices $U_{i j}$ and $V_{i j}$ such that

$$
U_{i j} A_{i} V_{i j}=D_{i}, \quad U_{i j} A_{j} V_{i j}=D_{j} .
$$

For all $k, A_{k}\left(A_{j}^{c t} A_{i}\right)=\left(A_{i} A_{j}^{c t}\right) A_{k}$.

Apply the $U_{i j}, V_{i j}$ and obtain

$$
U_{i j} A_{k}\left(A_{j}^{c t} A_{i}\right) V_{i j}=U_{i j}\left(A_{i} A_{j}^{c t}\right) A_{k} V_{i j}
$$

so

$$
U_{i j} A_{k} V_{i j} V_{i j}^{c t} A_{j}^{c t} U_{i j}^{c t} U_{i j} A_{i} V_{i j}=U_{i j} A_{i} V_{i j} V_{i j}^{c t} A_{j}^{c t} U_{i j}^{c t} U_{i j} A_{k} V_{i j}
$$

so

$$
\left(U_{i j} A_{k} V_{i j}\right)\left(D_{j}^{c t} D_{i}\right)=\left(D_{i} D_{j}^{c t}\right)\left(U_{i j} A_{k} V_{i j}\right) .
$$

Therefore, the matrix $U_{i j} A_{k} V_{i j}$ commutes with the nonscaler diagonal matrix $D_{j}^{c t} D_{i}$. Since the unitary transformation may be chosen so that like elements of $D_{j}^{c t} D_{i}$ appear together in order, $U_{i j} A_{k} V_{i j}$ is a direct sum of matrices of order $m<n$ for all $k$. Since the submatrices satisfy these conditions, the theorem is true by induction.

UNIVERSITY OF WISCONSIN 